\title{
Subculture human skeletal muscle cells to produce the cells with different Culture medium compositions
}

\author{
Ying Gao Balch \\ The University of Arkansas at Pine Bluff, Department of Human Sciences
}

Corresponding Author: Ying Gao Balch, The University of Arkansas at Pine Bluff, Department of Human Sciences

Received date: April 14 2021; Accepted date: April 27, 2021; Published date: April 30, 2021

Citation: Ying Gao Balch. (2021) Subculture human skeletal muscle cells to produce the cells with different Culture medium compositions. Clinical Research and Clinical Trials. 3(3); DOI: 10.31579/2693-4779/036

Copyright: (C) 2021 Ying Gao Balch, This is an open access article distributed under the Creative Commons Attribution License, which permits unrestricted use, distribution, and reproduction in any medium, provided the original work is properly cited.

\begin{abstract}
This study aimed to subculture human skeletal muscle cells (HSkMC) using a culture medium with different compositions to determine the most efficient medium for the growth of the human skeletal muscle cells. The culture media was divided into three groups: Group1. An HSkMC growth medium. Group 2. An HSkMC growth medium + with $10 \%$ high glucose $(\mathrm{GH})$. Group 3. An HSkMC growth medium $+10 \%$ fetal bovine serum (FBS). HSkMC from groups 1 to 3 gradually became round in shape and gathered in clusters. These changes differed between the groups. In group 3, the HSkMC clusters were more in numbers and gathered as significantly more prominent than in the other groups under the EVOS-Microscope shown. We concluded that by manipulating the composition of the culture medium, it is possible to induce HSkMC to promote the best growth.
\end{abstract}

Keywords: human skeletal muscle cells (hskmc); EVOS-Microscope

\section{Introduction:}

Evidence suggests that inflammation and apoptosis play a role in a skeletal muscle cell associated with diminished skeletal muscle cell function with healthy aging [1, 2-5]. Besides, Apoptotic signaling pathways can activate cysteine-dependent, aspartate-specific proteases (caspases-3), which are the end of proteases and integral to the final produced cell death [6-7]. Caspases-3 typically exists in an inactivated state in the cytoplasm, but it can be activated by proteolytic cleavage, and then hetero-dimerization and activates pro-caspase-3 start the caspase cascade $[8,9]$. Once the proteolytic cascade is switched on, it eventually cleaves and enables pro-caspase- 3 to induce the inflammation to the loss of skeletal muscle [9]. This study aimed to subculture human skeletal muscle cells (HSkMC) using culture media with different compositions to determine the most efficient medium for the growth of the human skeletal muscle cells to ensure that the research experiment more efficient.

\section{Materials and methods:}

Human skeletal muscle cells source: normal human skeletal muscle. Single donor: 52 years old black female and in the formulation of cryopreserved at the second passage in basal medium containing $10 \%$ FBS and 10\% DMSO (Cell Application, INC.).The Growth Medium at $4^{\circ} \mathrm{C}$ in the dark immediately upon arrival. Cryopreserved Vials have stored cry vials in a liquid nitrogen storage tank immediately upon arrival. To examine under a microscope to check if all the cells were attached to the bottom of the flask. Decontaminated the exterior of the cell culture flask with $70 \%$ alcohol. Placed the sealed flask in a $37^{\circ} \mathrm{C}, 5 \% \mathrm{CO}_{2}$ incubator for 2 hours as shipped. In a sterile Biological Safety Cabinet, opened the flask's cap very slowly and carefully, removed the transport medium by aspiration, and added fresh growth medium: $15 \mathrm{ml}$ for a T-75 flask. Placed the flask in a $37^{\circ} \mathrm{C}, 5 \% \mathrm{CO}_{2}$ humidified incubator with the loosened cap to allow gas exchange. To change the medium every other day.

Preparing cell culture flasks for culturing HSkMC: To ensure the Biological Safety Cabinet, with HEPA filtered laminar airflow, was in proper working condition. Clean the Biological Safety Cabinet with $70 \%$ alcohol to ensure it was sterile. It was turned the Biological Safety Cabinet blower on for $10 \mathrm{~min}$ before cell culture work. To make sure all serological pipettes, pipette tips, and culture medium were sterile. All of them follow the standard sterilization technique and safety rules. It should always be wearing gloves and safety glasses when working with human cells even though all the strains have been tested negative for HIV, Hepatitis B, and Hepatitis C. Handle all cell culture work in a sterile hood. 


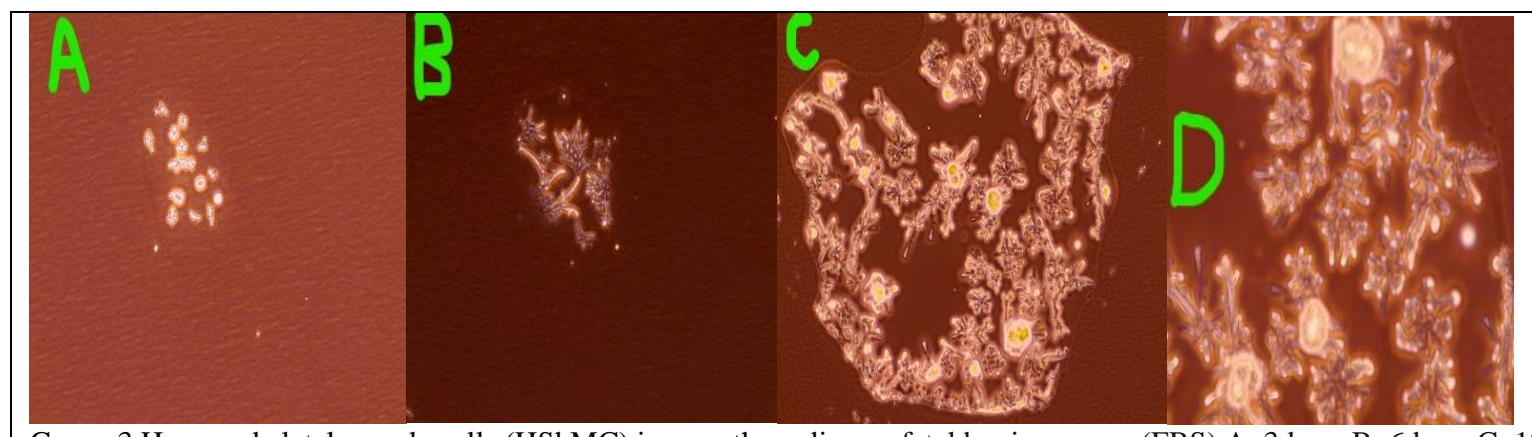

Group 3 Human skeletal muscle cells (HSkMC) in growth medium + fetal bovine serum (FBS) A: 3days, B: 6days, C: 10 Days, and 12 Days

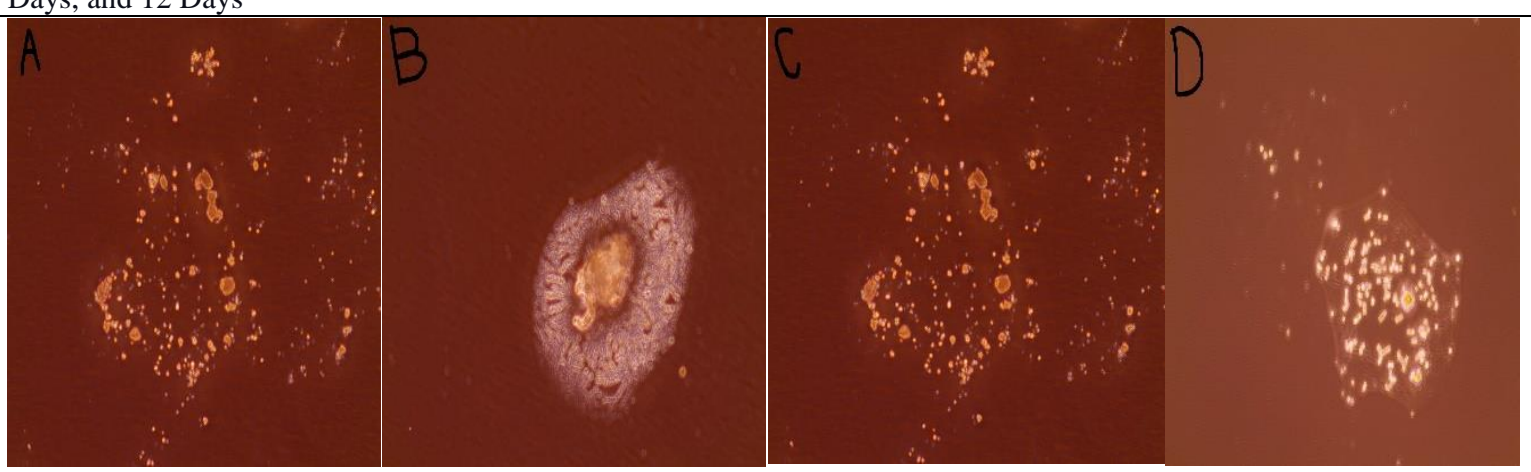

Group 2: Human skeletal muscle cells (HSkMC) in growth medium + high glucose (GH)

A: 3days, B: 6days, C: 10 Days, and 12 Days

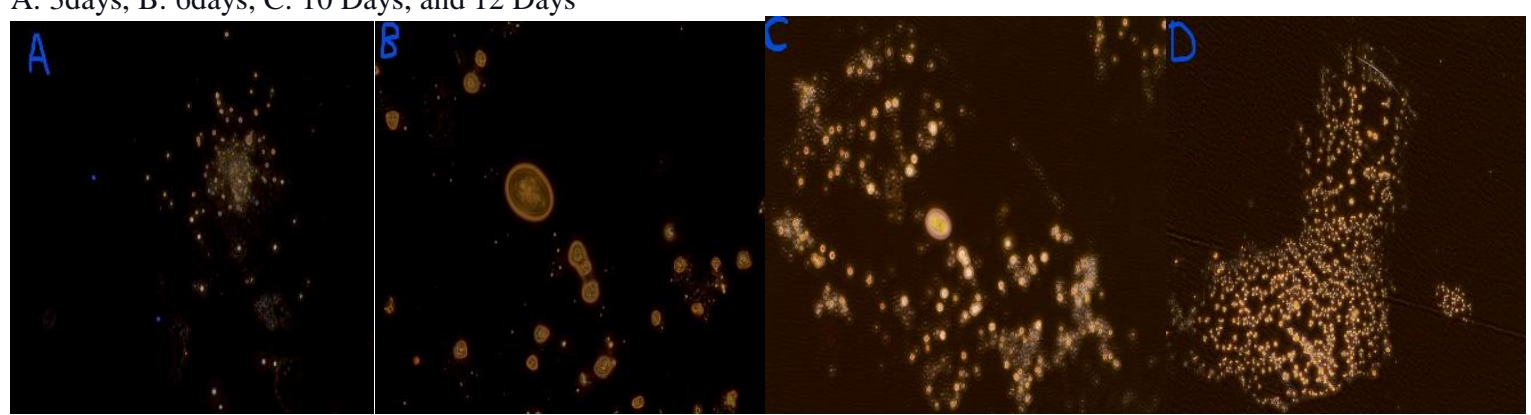

Group 1 Human skeletal muscle cells (HSkMC) Growth medium

A: 3days, B: 6days, C: 10 Days, and 12 Days

\section{Preparing subculture mediums:}

Took the Skeletal Muscle Cell Growth Medium from the refrigerator and decontaminated the bottle with $70 \%$ alcohol in a sterile hood. Pipetted 15 $\mathrm{ml}$ of Skeletal Muscle Cell Growth Medium into $20 \mathrm{ml}$ for each T-75 flask with different culture medium: Group 1. HSkMC growth medium. Group 2. HSkMC growth medium $+10 \%$ high glucose $(\mathrm{GH})$. Group 3. HSkMC growth medium $+10 \%$ fetal bovine serum (FBS). Placed the flask in a $37^{\circ} \mathrm{C}, 5 \% \mathrm{CO}_{2}$ humidified incubator with the loosened cap to allow gas exchange. To change the medium every other day. Water treated in a Milli-Q water purification system (TGI Pure Water Systems, USA). HSkMC growth medium, high glucose medium, and FBS of all acquired from (USA).

\section{Results:}

The three groups' culture medium, 1 to 3 , gradually became round in shape and gathered in clusters under the EVOS-Microscope shown. These changes differed between the groups. In group 3, the HSkMC clusters were more in numbers and gathered as bigger aggregates under EVOSMicroscopy shown. That groups 1 and 2 were similar in terms of the mean area of each aggregate; however, only in group 3, the number of aggregates and the total size of aggregates clusters were significantly more than in the other groups. We concluded that by manipulating the composition of the culture medium, it is possible to induce HSkMC to promote the best growth.

\section{Discussion}

HSkMC was capable of growing by manipulating the culture medium composition. In groups 1 to 3 , HSkMC got rounder increasingly during culture. Under the EVOS-Microscope shown, HSkMC was kept in culture media with a high glucose concentration without FBS; however, this characteristic led to a decreased cellular density, smaller formation, and aggregation of oval-round cellular clusters comparison with group 3. In group 3 exhibited the most significant results for aggregates clusters with observation under the EVOS-Microscope.

In all three groups in which essential medium was used as primary culture medium, it was observed the formation of cellular clusters; Group 2 was exposed to a stimulus with high glucose in the absence of FBS. FBS provides essential nutrients and growth factors to human skeletal muscle cells in culture and sustaining their proliferation [10, 11, 13]. Besides, FBS also has antioxidant properties, thus being critical for cellular survival. Therefore, groups 1, 2 of this study have observed the apoptotic effect caused without FBS [15]. High glucose concentration has been 
considered to be an effective inductor of the human cells. Still, some problems have been associated with its use throughout the culture. Some researchers have stated that a high concentration of glucose in culture media for a long time causes oxidative stress, cellular damage, early senescence, and apoptosis $[15,16]$. Thus, it is likely that the absence of antioxidant extrinsic factors, such as $\beta$-mercaptoethanol, might have contributed to a more significant cellular loss by oxidative stress in group 1. 2. This oxidative stress can be caused by a high glucose concentration for an extended period and the absence of FBS [12].

EVSO-Microscope analysis shown that in groups 1 and 2 in the ovalround cell clusters were less numerous and more isolated from each other than those in group 3 and 12 final days of group 3. Therefore, it was believed that the addition of extending days had promoted more efficiency by the effect of supplements like FBS during 12 days. Fetal bovine serum is composed of a mixture of hormones, growth factors, antibodies, and protein components responsible for maintaining cellularity in many cell cultures [14]. Moreover, Researchers believe that adding specific substances to the medium can promote human skeletal muscle cells' best growth at the proper culture mediums, like adding extrinsic factors, supplements, and a high glucose concentration.

\section{Reference}

1. Cortopassi GA, Wong A. (1999) Mitochondria in organismal aging and degeneration. Biochim Biophys Acta. 1410:183-193.

2. Higami Y, Shimokawa I. (2000) Apoptosis in the aging process. Cell Tiss Res. 301:125-132.

3. Anglade P, Vyas S, Hirsch EC, Agid Y. (1997) Apoptosis in dopaminergic neurons of the human substantia nigra during normal aging. Histol Histopathol. 12:603-610.

4. Phaneuf S, Leeuwenburgh C. (2001) Apoptosis and exercise. Med Sci Sports Exerc. 33:393-396.

5. Pollack M, Phaneuf S, Dirks A, Leeuwenburgh C. (2002) The role of apoptosis in the normal aging brain, skeletal muscle, and heart. Ann NY Acad Sci. 959:93-107.

6. Thornberry NA. (1999) Caspases: a decade of death research. Cell Death Diff. . 6:1023-1027.
7. Sun XM, MacFarlane M, Zhuang J, Wolf BB, Green DR, Cohen GM. (1999) Distinct caspase cascades are initiated in receptormediated and chemical-induced apoptosis. J Biol Chem. 274:5053-5060.

8. Sandri M, Carraro U, Podhorska-Okolów M, et al. (1995) Apoptosis, DNA damage and ubiquitin expression in normal and MDX muscle fibers after exercise. FEBS Lett. . 373:291-295.

9. Dirks A, Leeuwenburgh C. (2001) Apoptosis in skeletal muscle with aging. Am J Physiol Regular Integr Comp Physiol. 282:R519-R527.

10. Meuleman N, Tondreau T, Delforge A, M. Dejeneffe, et al. (2006) Human marrow mesenchymal stem cell culture: serum-free medium allows better expansion than classical $\alpha$-MEM medium Eur J Haematol, 76 , pp. 309-316

11. Jung S, Sen A, Rosenberg L. (2012) Human mesenchymal stem cell culture: rapid and efficient isolation and expansion in a defined serum-free medium J Tissue Eng Regen Med, 6, pp. 391403

12. Janjic C, Wollheim C.B. (1992) Effect of 2-mercaptoethanol on glutathione levels, cystine uptake and insulin secretion in insulinsecreting cells. Eur J Biochem, 210, pp. 297-304

13. BerlierJ.L, Kharroubi I, Zhang J, Mathieu M, et al. (2015) Glucose-dependent insulinotropic peptide prevents serum deprivation-induced apoptosis in human bone marrow-derived mesenchymal stem cells and osteoblastic cells Stem Cell Rev Rep, 11, pp. 841-851

14. Cho H, Lee A, Kim K. (2018) The effect of serum types on Chondrogenic differentiation of adipose-derived stem cells Biomater Res, 22 , pp. 1-10

15. Li H, Lam A, Kim Chung S. (2010) High dosage of Exendin-4 increased early insulin secretion in differentiated beta cells from mouse embryonic stem cells Acta Pharmacol Sin, 31 , pp. 570-577

16. Huang L.S, Jiang M.H, Wu H.L, J. (2010) Chen, Huang Y, et al. Implantation of bFGF-treated islet progenitor cells ameliorates streptozotocin-induced diabetes in rats Acta Pharmacol Sin, 31, pp. $1454-1463$ 